\section{The new classification}

\section{of periodontal}

\section{diseases}
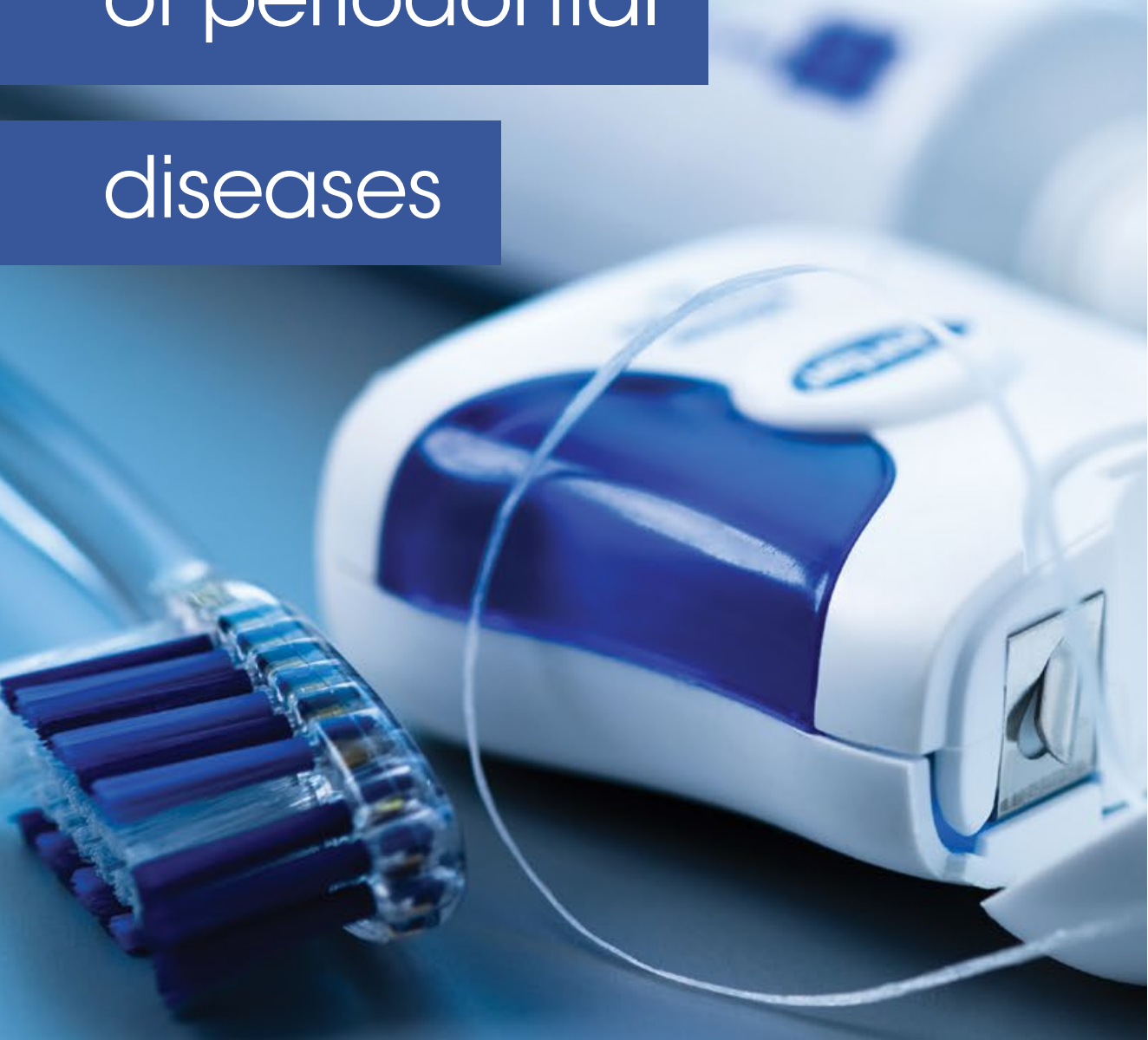

\section{Author information}

'Ammar Ahmed Zaki BDS (LIV), MSci (KCL), MFDS (RCSEd) completed a four-year MSci in integrated Physiology and Pharmacology for Research from King's College London in 2013. He went on to complete his $B D S$ from the University of Liverpool in 2018. He will be completing his $P G$ Cert in teaching and learning in October 2020 and has passed the MFDS exams. He is currently carrying out his second year as a Dental Core Trainee in OMFS in North Wales. He is interested in pursuing a career in restorative dentistry and hopes to apply for speciality training in the coming years.

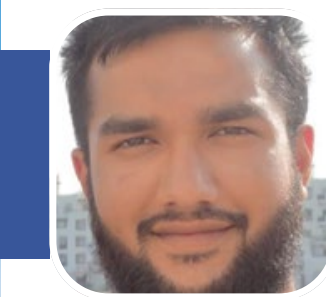

Ammar

Ahmed

Zaki $^{1}$

explains

the newly

redesigned periodontal

disease classification

framework for the

benefit of the whole

dental team.

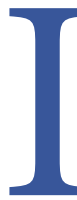

n 2017 the American Academy of Periodontology (AAP) and European Federation of Periodontology

(EFP) co-presented the new

classification for periodontal and peri-implant diseases and conditions at the world workshop. ${ }^{1}$ This was to replace the Armitage 1999 classification of periodontal diseases, which had been used for nearly two decades. ${ }^{2}$ This new redesigned periodontal disease classification framework included a multi-dimensional staging and grading system, the inaugural classification for peri-implant diseases and conditions and a recategorisation of various forms of periodontitis. ${ }^{1}$ Many of those who were first introduced to the new system felt it was only suitable for use by researchers and specialists, thereby excluding dental therapists, dental hygienists and general dental practitioners, who treat a significant number of the periodontal disease cases.

The new worldwide classification was based on interproximal clinical attachment loss. ${ }^{1}$ This is due to clinical attachment loss being the gold standard for diagnosis and monitoring of periodontal disease. ${ }^{3}$ However, within the United Kingdom, for example, when a patient has a BPE of 4, we would go on to carry out a pocket probing depth chart and don't routinely record clinical attachment loss, making it difficult to implement the new classification. In 2018 the British Society of Periodontology (BSP) published a revised version of the 2017 classification to help steer practitioners through to diagnosing a patient using the 2017 classification system in a pragmatic way for general practice. ${ }^{4}$ This revised version is based mainly on radiographic bone loss ${ }^{5}$ and a flowchart was developed by BSP to aid NHS and private practitioners with implementing the 2017 classification. ${ }^{4}$ 
The new classification brought about a few changes. The first change and one of the most profound is that it defined and recognised periodontal health for the first time. ${ }^{4}$ This was key as it meant many people who had gone without any periodontal diagnosis for many years after an examination would now have one. Recognising periodontal health ultimately allows for better lines of communication and a point of comparison if the patient ever develops periodontal disease in the future.

The extent of the disease is the same as before, with localised being less than $30 \%$ of the teeth being involved, and generalised, if it is more than $30 \%{ }^{4}$ However, there has been a reintroduction of molar/incisor pattern, which was the defining factor for juvenile periodontitis in periodontal classification used before Armitage 1999. ${ }^{6}$ The new classification also replaced the terms 'chronic' and 'aggressive' used in the Armitage model as periodontitis is a broad spectrum of a single disease, and not two distinct ones. There was also the addition of staging and grading the disease. ${ }^{4}$ Staging refers to the severity and is based upon the percentage of bone loss compared with the root length. ${ }^{4}$ The site with the worst amount of bone loss within the mouth due to periodontitis is used for this. The grading, however, determines the susceptibility of the patient to periodontitis. It predicts the presence of disease in the absence of treatment by comparing the percentage of bone with the patient's age. ${ }^{4}$ The terms 'staging' and 'grading' were taken from the model used to classify cancers and so some sensitivity needs to be used when discussing this with patients.

Evidence-based risk factors including smoking, sub-optimally controlled diabetes, pregnancy, stress, genetics etc, also need to be noted. ${ }^{4}$ This is a great addition as it allows clinicians to discuss these risk factors with the patient which could result in lifestyle changes and greater control of a patient's disease progression. The new classification means that clinicians can assess quickly at what stage their patient is in their pathway to achieving periodontal health by simply looking at the diagnosis.

Peri-implant disease also had its first appearance in the new classification ${ }^{5}$ and with the rise in the number of patients who have implants this was an important step to take. It allows clinicians to identify and therefore manage appropriately periodontal diseases and conditions associated with the placement of implants. These include peri-implant health, peri-implant mucositis and implantitis as well as hard tissue deficiencies. ${ }^{7}$

\section{'Evidence based risk factors including smoking, sub-optimally controlled \\ diabetes, pregnancy, stress, genetics etc, also need to be noted.'}

It is crucial to remember that although the classification may have changed, the management is still the same. ${ }^{7}$ This includes carrying out bleeding on probing (BOP) and periodontal pocket depth (PPD) checks on patients who have been identified as having periodontal disease whether it is stable, in remission or currently unstable. ${ }^{7}$ Patients who then need treatment including supra and subgingival scaling and other nonsurgical periodontal therapy (NSPT) should also be able to access this treatment.

The prevalence of severe periodontal disease worldwide ranges from between $10-15 \% .{ }^{8}$ This is especially crucial as unfortunately litigation relating to mismanagement and misdiagnosis of periodontal disease continues to rise and the results to both the patient and the clinician can be devastating. ${ }^{9}$ When considering the top claims by value within the United Kingdom in 2015, 5.5\% involved implants and periodontal disease, $28.8 \%$ involved just implants and $44.7 \%$ involved periodontal disease. ${ }^{9}$ The periodontal legal claims most frequently involved failure to diagnose by taking adequate radiographs to assess bone levels, evidence of risk assessment, monitoring or treatment of the disease. ${ }^{9}$ There are increasing claims for failure to offer specialist care and a failure to refer appropriately. ${ }^{9}$

Many simple steps can be implemented to avoid mismanagement of periodontal disease and litigation including explaining the diagnosis to the patient, constructing a long-term plan with the patient and documenting it. It is also important that, if working under a prescription by a dentist, the prescription is adequate. If this is not the case, then it should be discussed with the prescribing dentist prior to commencement of management and treatment. The new classification allows the ability to reach a diagnosis and lets the clinician know exactly where the patient is within the disease cycle, and this can help grant patients the best possible treatment for them.

\section{References}

1. American Academy of Periodontology. Proceedings from the 2017 World Workshop on the Classification of Periodontal and Peri-implant Diseases and Conditions. 2017. Available at: https:// www.perio.org/2017wwdc (accessed September 2020).

2. Armitage G C. Development of a classification system for periodontal diseases and conditions. Ann Periodontol 1999; 4: 1-6.

3. Gupta N, Rath S K, Lohra P. Comparative evaluation of accuracy of periodontal probing depth and attachment levels using a Florida probe versus traditional probes. Med J Armed Forces India 2015; 71: 352-358.

4. British Society of Periodontology. Implementing the 2017 Classification of Periodontal Diseases to Reach a Diagnosis in Clinical Practice. 2018. Available at: https://www.bsperio.org.uk/assets/ downloads/111_153050_bsp-flowchartimplementing-the-2017-classification.pdf (accessed September 2020).

5. Dietrich T, Ower P, Tank M et al. Periodontal diagnosis in the context of the 2017 classification system of periodontal diseases and conditions - implementation in clinical practice. Br Dent J 2019; 226: 16-22.

6. Highfield J. Diagnosis and classification of periodontal disease. Aust Dent J 2009; 54(Suppl 1): S11-26.

7. Ower P. New classification system for periodontal and peri-implant diseases. Dent Update 2019; 46: 8-11.

8. Meyer M S, Joshipura K, Giovannucci E, Michaud D S. A review of the relationship between tooth loss, periodontal disease, and cancer. Cancer Causes Control 2008; 19: 895-907.

9. Reena W. Reena's Notes: How to Avoid Periodontal Litigation in General Dental Practice with Dr Ian Dunn. 2018.

Available at: https://www.reenawadia.com/ reenas-notes-how-to-avoid-periodontallitigation-in-general-dental-practice-withdr-ian-dunn/ (accessed September 2020).

https://doi.org/10.1038/s41407-020-0435-5 